\title{
Das Blutdruckmedikament kommt mit der Drohne
}

\section{Rudolf Stumberger}

PD Dr. phil., Soziologe und Publizist, Münchner Pressebüro

\begin{abstract}
Drohnen werden nur im Militär eingesetzt? Mitnichten. Immer öfter finden die unbemannten Flugsysteme auch im zivilen Bereich Anwendung. Diesen Sommer hat die Schweizer Post Testflüge mit Paketdrohnen im Berner Seeland gestartet. Nicht nur für die Transportbranche, sondern auch für die medizinische Versorgung würde der Einsatz von Paketdrohnen eine grosse Veränderung bedeuten.
\end{abstract}

Für Amalie und Hubertus K. war er endlich da, der langersehnte Urlaub in den Schweizer Bergen. Einquartiert hatte sich das Ehepaar in einem Wellness-Hotel mit

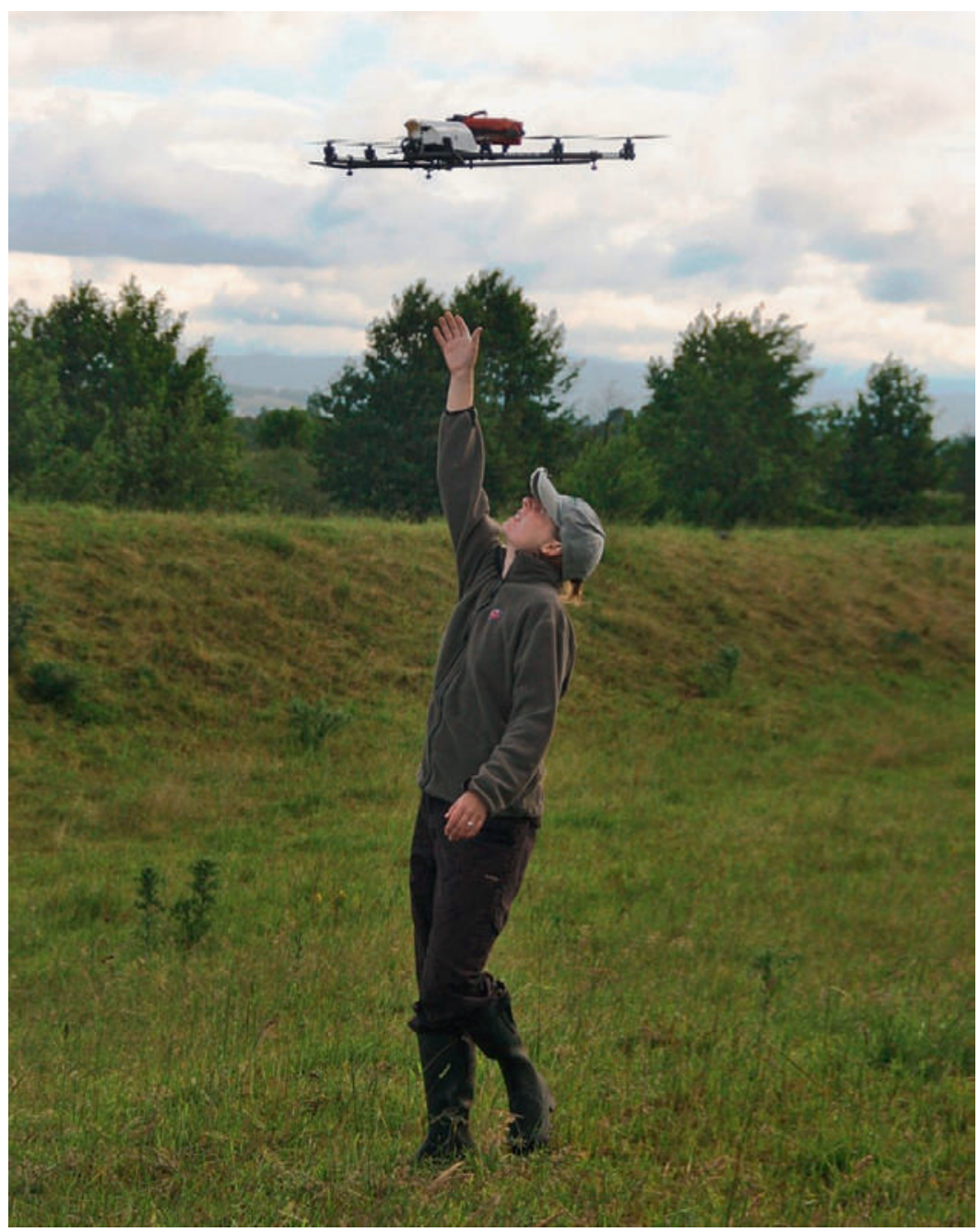

Drohnen sollen helfen, die medizinische Versorgung in der Schweiz zu verbessern.
Gipfelblick und mit dabei hatte man alles, was notwendig war: zum Beispiel die Tabletten zur Senkung des Blutdruckes für Herrn K. Die blieben freilich im Badezimmer zurück, wenn das Ehepaar tagsüber seine Ausflüge mit dem Auto unternahm. Zum Beispiel in das abgelegene Bergtal in der Ostschweiz. Nur, an diesem Tag gab es kein Zurück. Eine Mure war am Nachmittag abgegangen und hatte die Zufahrtsstrasse zu dem Bergdorf unpassierbar gemacht. Zusammen mit anderen abgeschnittenen Touristen mussten Herr und Frau K. im Dorf eine Notunterkunft beziehen. Freilich: Die Räumung der Strasse konnte zwei oder drei Tage dauern und Herr K. sorgte sich ohne Tabletteneinnahme um seinen Blutdruck. Schlechtes Wetter machten Hubschrauberflüge unmöglich. Dann kam die Hilfe doch aus der Luft: Mit einem surrenden Geräusch setzte am nächsten Mittag eine Paketdrohne auf dem Friedhof des Bergdorfes auf. Mit in der Fracht dabei der blutdrucksenkende Wirkstoff für Herrn K.

So ähnlich könnte das Szenario aussehen, für das derzeit die Schweizer Post Testflüge mit sogenannten $\mathrm{Pa}$ ketdrohnen unternimmt. Drohnen sind unbemannte Flugsysteme, die neben der militärischen Nutzung zunehmend auch im zivilen Bereich Verwendung finden: von der Vermessungstechnik über die Inspektion von Gebäuden oder Öl-Plattformen im Meer bis hin zu Objektbefliegungen in Sachen Denkmalschutz oder Anwendungen in der Landwirtschaft. So setzte man in einem Versuchsprojekt etwa Drohnen ein, um mit Hilfe von Wärmekameras sich im Getreide versteckende Rehkitze aufzuspüren und sie so vor dem Tod durch den Mähdrescher zu bewahren. Und es war ein wirklicher Marketing-Knaller, als der Versandhändler Amazon im Dezember 2013 ankündigte, er wolle in Zukunft seine Pakete mit einer Lastendrohne ausliefern. 


\section{Verhelfen Drohnen der Transportbranche zu einem "Quantensprung»?}

Eine Herausforderung für traditionelle Paketzusteller wie der Post. Für Dieter Bambauer, Logistik-Chef der Schweizer Post, könnte es durch den Einsatz von sogenannten Transportdrohnen gar zu einem «Quantensprung" in der Transportbranche kommen. «Die $\mathrm{Zu}$ nahme im Paketgeschäft durch den Onlinehandel und der gleichzeitige Druck auf die Gewinnmargen treibt uns an», so seine Begründung für die Testflüge rund um Bern.

\section{Die dringliche Auslieferung von Medikamen- ten ist prioritär bei der Erprobung von Paket- drohnen durch die Schweizer Post.}

Die Auslieferung von dringend benötigten Medikamenten steht auch an vorderster Stelle bei der Erprobung von Paketdrohnen durch die Schweizer Post. «Wir prüfen den Transport von Arzneimitteln in abgelegene Häuser oder in Siedlungen, die etwa durch einen Erdrutsch nicht mehr zugänglich sind», sagt Postsprecher Bernhard Bürkli. So könnten auch von der Aussenwelt abgeschnittene Personen, die zum Beispiel auf Blutdruckmedikamente angewiesen sind, versorgt werden. In Zusammenarbeit mit Swiss World Cargo hat die Schweizer Post diesen Sommer einen Feldversuch im Berner Seeland gestartet, dabei handelt es sich um ein eher flaches Gelände. Durchgeführt wurden die Testflüge mit einer Paketdrohne der kalifornischen Firma Matternet, die Lasten von 1 bis 20 Kilo tragen kann. Die Firma hat bereits Tests in Ländern mit wenig entwickelter Infrastruktur wie Bhutan und Haiti durchgeführt. Dieter Bambauer, Chef der Schweizer Post-Logistik, sieht drei Anwendungsbereiche für Paketdrohnen: Sie könnten Randregionen beliefern, Menschen in Notsituationen erreichen und dringende Spezialsendungen wie Laborproben erledigen. Bambauer: «Wir starten die Lieferungen im medizinischen Feld.» Jetzt im Herbst sollen Testflüge in den Bergen des Berner Oberlandes beginnen.

\section{Verschärfte gesetzliche Bestimmungen}

Ein zentrales Moment bei der Anwendung von Drohnen sind dabei die gesetzlichen Regeln. Das Bundesamt für Zivilluftfahrt (BAZL) hatte im August vergangenen Jahres diese Regeln verschärft: «In Zukunft dürfen Drohnen oder Flugmodelle in einer Entfernung von weniger als 100 Metern von Menschenansammlungen nur mit einer Bewilligung des Bundesamtes für Zivilluftfahrt betrieben werden.» Mit der geänderten Verordnung reagiert die Behörde auf die Besorgnis der Bevölkerung über die wachsende Zahl von Drohnen am Himmel in der Schweiz. Generell unterliegt der Einsatz von Flugmodellen oder Drohnen mit einem Gewicht von über 30 Kilogramm oder mit Steuerung ohne direkten Augenkontakt bereits heute einer Sonderbewilligung durch das BAZL.

Diese Steuerung ohne Sichtkontakt aber ist die zentrale Voraussetzung für einen künftigen Einsatz von Transportdrohnen etwa zum Medikamententransport im Notfall. Denn bei Flügen über Langstrecken von mehreren Kilometern ist dieser Sichtkontakt nicht möglich, die unbemannten Fluggeräte fliegen dann mit einer GPS-Steuerung. Ausprobiert hat dies auch die Deutsche Post, die ebenso wie die Schweizer die künftigen Möglichkeiten von Paketdrohnen testen. Im Okto-

Im vergangenen Oktober wurde erstmals eine Apotheke auf der deutschen Nordseeinsel Juist durch eine Paketdrohne beliefert.

ber vergangenen Jahres wurde zum ersten Mal in Deutschland eine Apotheke auf der Nordseeinsel Juist durch eine Paketdrohne mit Medikamenten beliefert. Der Paketkopter flog vollautomatisch und ohne Sichtkontakt durch einen Piloten die Strecke von zwölf Kilometern zwischen der Stadt Norden auf dem Festland und der Inselgemeinde. Die Hauptaufgabe der Drohne: Arzneimittel auch dann auf die Insel zu bringen, wenn keine alternative Infrastruktur per Flugzeug oder Schiff zur Verfügung steht. Die Tests werden jetzt ausgewertet. 